\title{
A Dedicated Multi-Beam SEM for Transmission Imaging of Thin Samples
}

Pieter Kruit $^{* *}$ and Wilco Zuidema ${ }^{1}$

1. Delft University of Technology, Department of Imaging Physics, Delft, The Netherlands.

* Corresponding author: p.kruit@tudelft.nl

Fast, large area imaging in combination with a large computer memory and modern image recognition techniques is opening up a new way of doing microscopy, which could be referred to as "Google map microscopy".

In a few successive PhD projects, we transformed a FEI Nova-nano SEM column into an instrument with an array of 14x14 focused electron beams on the sample [1-3]. The original source module was replaced by one in which the beam from the Schottky source is divided into 196 separate sub-beams. Each beam has about $1 \mathrm{nA}$ of current and the same brightness as the original single beam. The array of source images is subsequently demagnified by the existing SEM optics to an array with a pitch of typically 0.5 to 5 microns. In scanning electron microscopy, images are usually formed by collecting the secondary electrons (SE) or the backscattered electrons (BSE). In a multi-beam system, it is a real challenge to keep the secondary and backscatter electrons from the separate beamlets apart and guide them to an array of individual detectors. We have managed to do that for the SE signal, but still suffer from cross-talk between the beams. Zeiss developed a commercially available multi-beam scanning electron microscope [4] following the same principles, which routinely collects large area images.

We have also made images with the transmission signal. Tissue sections with a thickness between 50 and $200 \mathrm{~nm}$ are placed directly on top of a scintillator. The array of light signals is imaged onto a detector array $[5,6]$, see figure 1 . This gives surprisingly high contrast images, comparable to the best BSE images, see figure 2. In fact, for thin biological sections, BSE or transmission imaging is preferable over SE imaging because SE imaging relies primarily on topological contrast, while BSE and transmission imaging shows the staining of the sample.

With these new insights, we are now developing a very much simplified system in cooperation with industrial partners. The new MBSEM is based on a Thermo Scientific Apreo SEM, figure 1. Using the original lenses, a grid of $8 \times 8$ electron beams with a current of 0,2 to $1 \mathrm{nA}$ are is focused to spots as small as $8 \mathrm{~nm}$ on the sample. The stage with light objective is based on a Delmic Secom system, which adds an optical microscope to the electron microscope. Technolution BV develops an arrayed multi-pixel photon counter and storage buffer. There is no multi-beam SE detection, but the microscope can also be operated in single beam mode with the regular detectors [7].

References:

[1,2,3] Y. Zhang, A. Mohammadi-Gheidari and Y. Ren, PhD theses Delft University of Technology.

[4] A.Eberle et al., J Microsc. 259 (2015), p. 114.

[5] AC Zonnevylle et al., J Microsc. 251 (2013), p. 58.

[6] Y Ren and P Kruit, Journal of Vacuum Science \& Technology B, 34.6 (2016), p. 06KF02.

[7] This work is a cooperation between Delft University, Thermo Fischer Scientific, Delmic and

Technolution. 

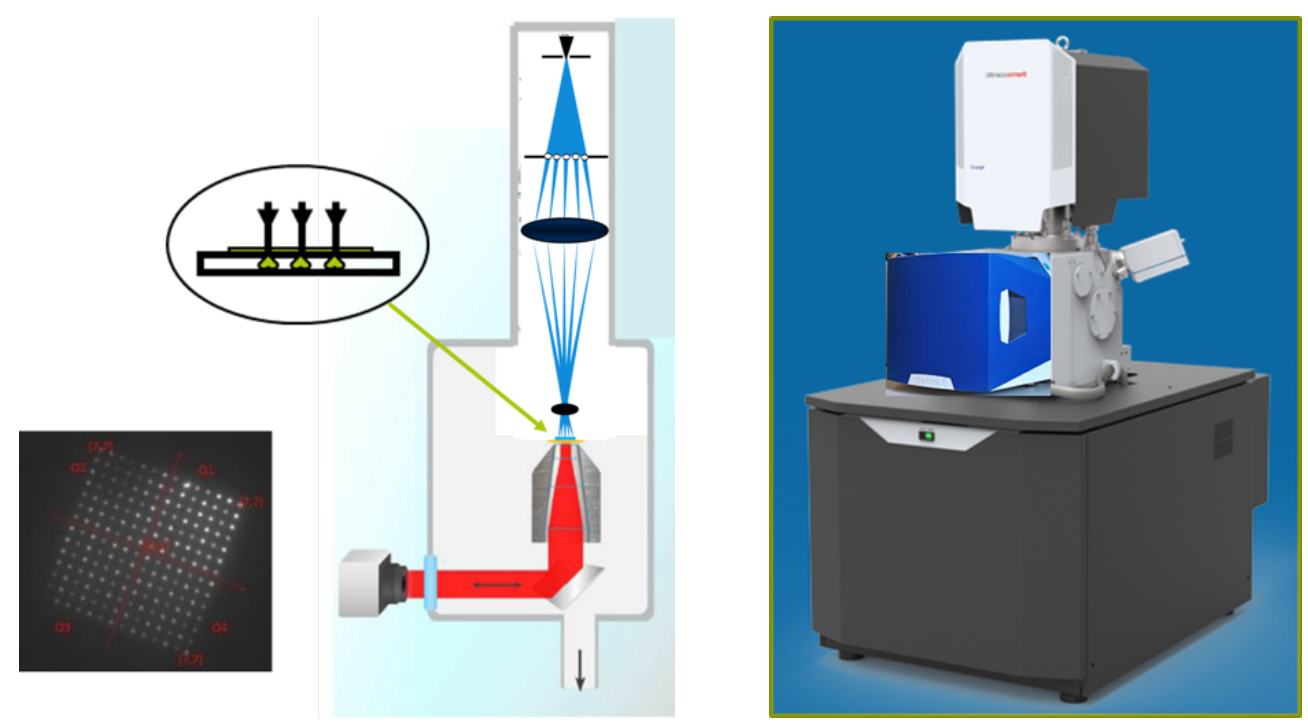

Figure 1. Schematic cross section of the dedicated Multi-Beam SEM for transmission imaging of thin samples. Right: Artist impression of the small multi beam SEM.

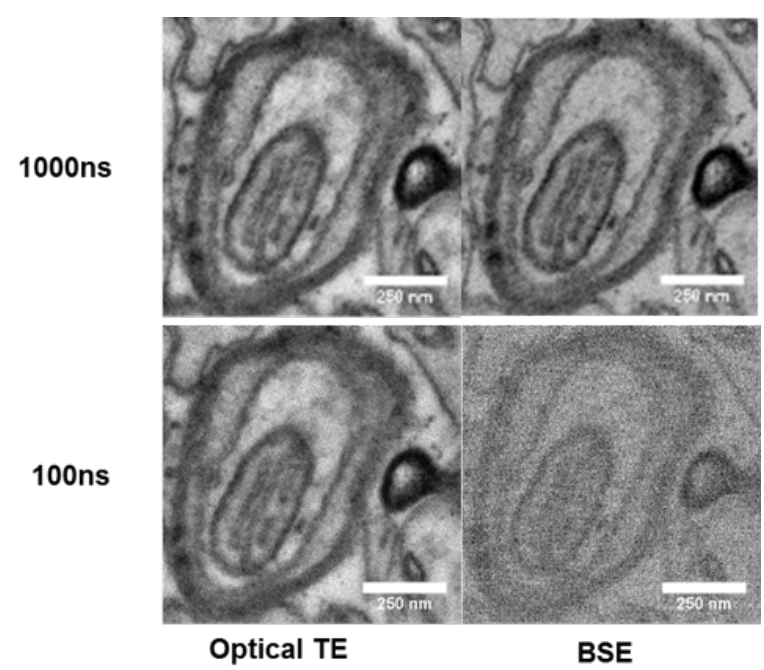

Figure 2. Image quality of transmission imaging versus backscatter imaging (4keV, 800pA). 